\title{
Efficient Calibration and Recalibration of Metal Oxide Gas Sensors by a New Mathematical Procedure
}

\author{
Rolf Seifert, Hubert B. Keller \\ Institut für Angewandte Informatik (IAI), Forschungszentrum Karlsruhe, \\ Hermann-von-Helmholtz-Platz 1, D-76344 Eggenstein-Leopoldshafen \\ Kevin Frank, Heinz Kohler \\ Institut für Sensorik und Informationssysteme (ISIS), Hochschule Karlsruhe - Technik und Wirtschaft, \\ Moltkestr. 30, 76133 Karlsruhe
}

\section{Introduction}

There is a growing need of economic online and in-situ field analysis applications like discriminated monitoring of toxic gas leakages, online monitoring of volatile components in chemical and biochemical processes, quality monitoring in food processing, etc. In this context, the isothermally operated metal oxide gas sensors (MOGs) with tin oxide as base material are manifold introduced due to their high sensitivity, long-term stability and low price. Their sensitivity to specific gas components, however, cannot be cultivated with high discrimination to others. On the other hand, it could be shown $/ 1,2,3,4 /$ that by periodic variation of the sensor temperature and simultaneous sampling of the so called Conductanceover-Time-Profiles (CTPs), by appropriate choice of sensor additives to the tin oxide material and by using an innovative mathematical calibration and evaluation procedure, valuable signal information can be extracted to be numerically analysed for substance identification and concentration determination. Although MOGs are very low-priced with respect to production and operation, they show, nevertheless, the disadvantage that for more accurate monitoring tasks every individual sensor element has to be calibrated. This is very time consuming and expensive. For a mono-component analysis, a single sensor element typically has to be measured for calibration at about five calibration points, i.e. dosed concentrations of the gas under consideration have to be adjusted, and the resulting signal patterns have to be sampled.

These extraordinary expenses for calibration are a consequence of unavoidable production inaccuracies of the sensor elements which lead to unreproducibilities of the gas analytic attributes. Therefore, although the signal patterns of the various sensor elements of a production batch are quite similar, each sensor element (SE) has to be costly calibrated, in order to yield high analytic performance. The same is true if a sensor chip has to be exchanged in case of a defect or simply has to be recalibrated.

\section{Basic idea of the new calibration procedure}

The idea of the new calibration procedure is, to use the similarity of signal patterns of the various sensor chips of a production batch. The main differences of such signal patterns are a shift (additive term) and an elongation (linear term) in conductance, whereas the time locations of the peaks remain more or less unchanged. This can be seen in Fig. 1, where the signal patterns (CTPs) of four sensor chips, investigated in more detail in $/ 5 /$, are plotted when exposed to $500 \mathrm{ppm}$ CO in synthetic air. These sensitive layers were prepared with variations in thickness of the sensitive layer on less defined interdigitated electrode geometries, both prepared by thick-film micro-dispensing methods, to simulate preparation tolerances within a batch. The same change of the CTP can be observed in the case of a "drifting" sensor. Drifting sensor means that the sensor signals, measuring the same gas probe, are changing in the course of time, due to the aging processes of the sensor element.

The striking advance of the new calibration procedure is that only the CTPs of one single SE , the socalled class reference element of the batch, have to be sampled at all calibration points. Every other SE of the batch is measured at only one calibration point (the so-called reference calibration point). Next, for every SE a mathematical function (the so-called approximation function) is determined which in a best manner fits the signal pattern of the reference calibration point using the class reference element to the signal pattern of the same calibration point yield by the corresponding SE. This means, if, for example, 
sensor 1 in Fig. 1 is the class reference element and the reference calibration point is 500 ppm, that an approximation function for sensor2 has to be determined which maps the CTP of sensor1 to the related CTP of sensor2 in a best manner. This means that each single point of the CTP of sensor1 is mapped to the related point of the calculated CTP of sensor2 in such a manner that, for example, the root of the sum of quadratic differences of the calculated points and sampled points of the CTP of sensor2 is minimal. This action is repeated for all other sensor chips of the production batch, i.e. in our case for sensor3 and for sensor4.

With these approximation functions the signal patterns of the class reference element are transferred for all other calibration points to yield signal patterns for all other SE of the batch.

The result of this procedure is that appropriate CTPs for all SE at all calibration points are available, although only the class reference chip has to be measured at all calibration points. The other SE of the batch have to be measured only at the reference calibration point. Because five calibration points for each sensor chip are typically necessary, this calibration procedure can reduce the above mentioned calibration expenses essentially by the factor of five for all SE of the batch, which are not the class reference element.

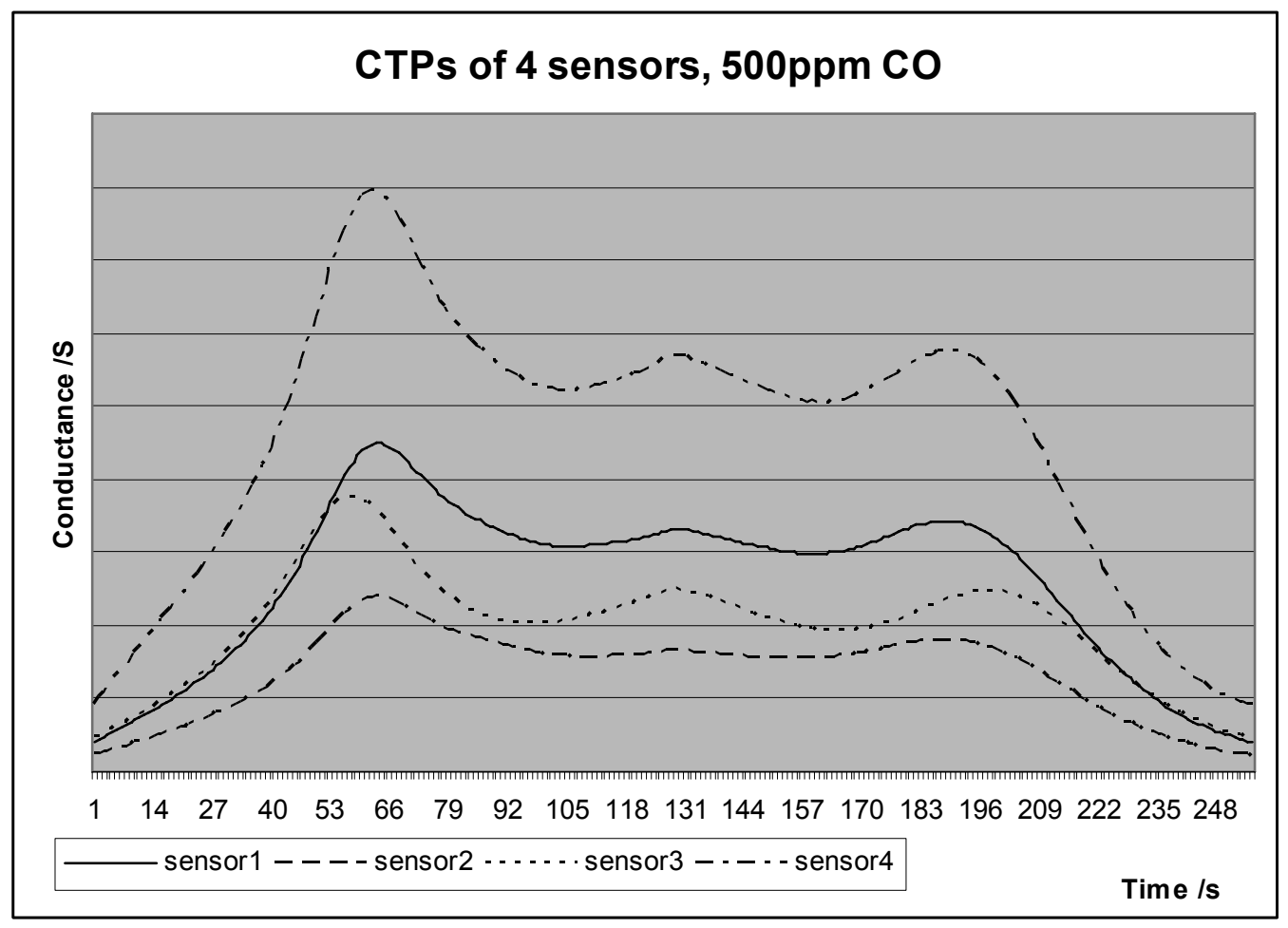

Fig. 1: CTPs sampled at 500 ppm CO in synthetic air on four sensors of the same batch. The CTPs represent one period of thermocyclic triangular temperature variation in the range $100^{\circ} \mathrm{C} \leq \mathrm{T} \leq 420^{\circ} \mathrm{C}$

\section{Comparison of measured CTPs and approximated CTPs}

To demonstrate the effectiveness of the new procedure the CTPs sampled on the four sensors when exposed to $\mathrm{CO}$ in synthetic air $\left(50 \% \mathrm{rH}\right.$ at $\left.22^{\circ} \mathrm{C}\right)$ are compared to the calculated ones. The calibration points in this example are 0ppm, 250ppm, 500ppm, 1000ppm and 2000ppm CO. Sensor1, see again Fig. 1 , is chosen as class reference element. This means, sensor1 is measured at all calibration points, the other SE are sampled only at the reference calibration point at 500ppm CO. Fig. 1 shows the signal patterns (in this example CTPs) of the four sensor chips at this calibration point at 500ppm. Using the calculated approximation functions, the approximated CTPs of the other SE are determined for all calibration points. For evaluation of this method, Fig. 2 und Fig. 3 show the measured CTP and the approximated CTP of an other SE, in this example sensor2, at 500ppm CO and at 1000ppm CO. It can be clearly seen that in both cases the approximated CTPs are close to the measured ones. Further investigations gave evidence that this method of approximation is also suitable for sensor3 and sensor4 as well. 


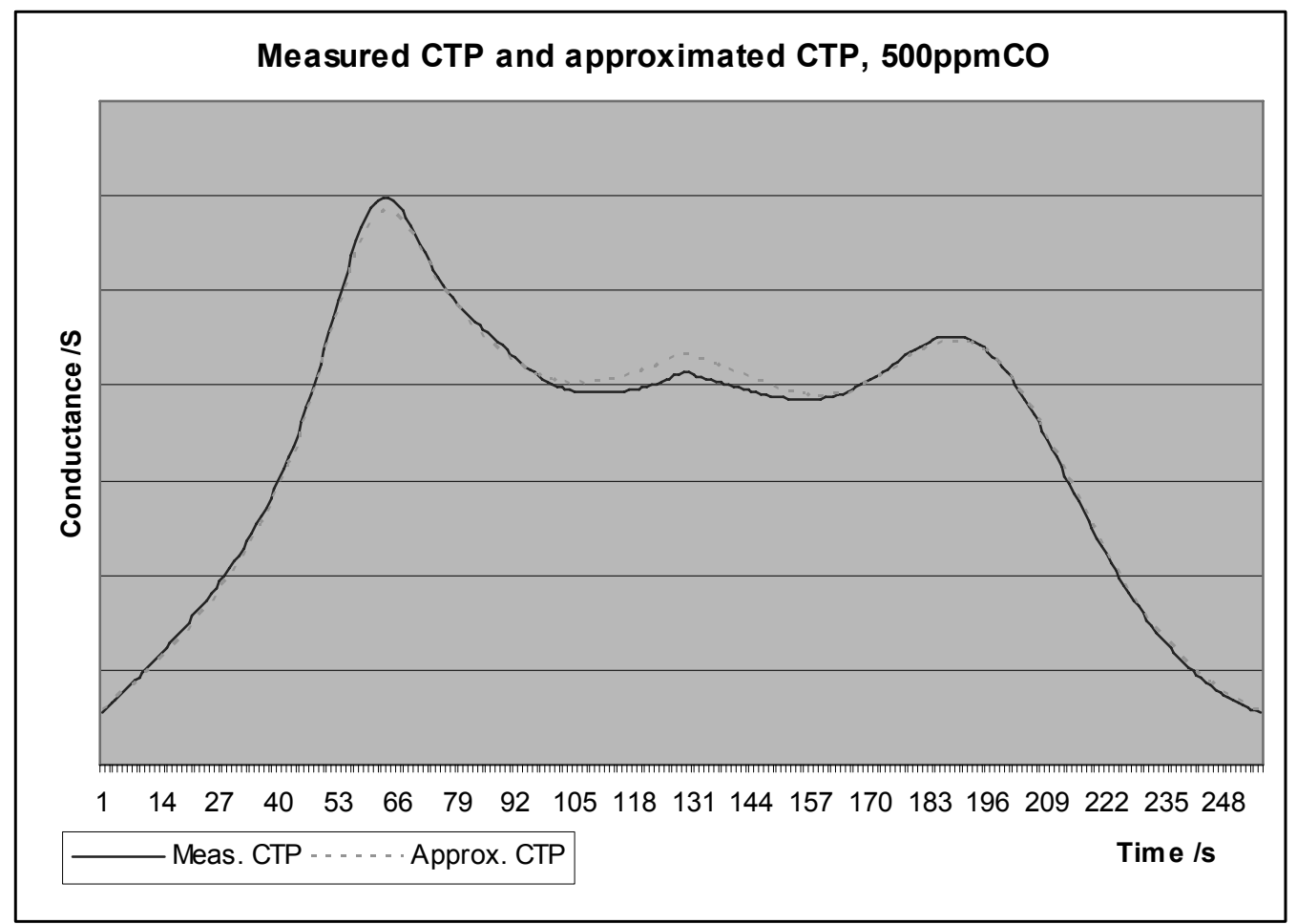

Fig. 2: Comparison between measured CTP and approximated CTP of sensor2 at $500 \mathrm{ppm} \mathrm{CO}$ in synthetic air.

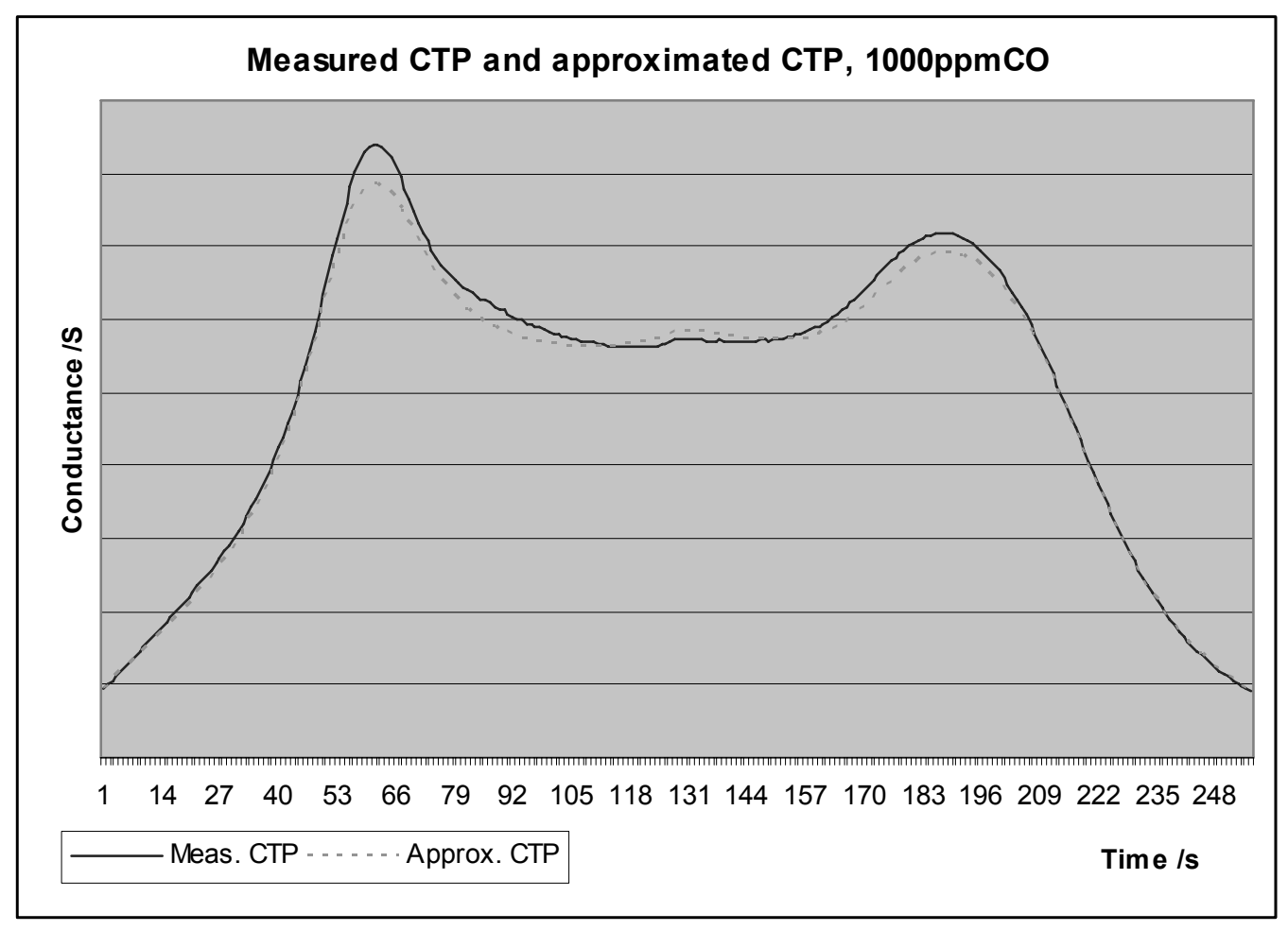

Fig. 3: Comparison between measured CTP and approximated CTP of sensor2 at 1000 ppm CO in synthetic air. 


\section{Analysis of $\mathrm{CO}$ in synthetic air}

In the final step of this investigation, the calibration data set of sampled and approximated CTPs as introduced above for sensor2, is now used to determine the $\mathrm{CO}$ concentrations from a new data set sampled at different gas mixtures (dosed) in the measurement mode. For this analysis, the mathematical calibration and evaluation procedure as described in $/ 6 /$, is used. The results of the analysis are given in Table 1 and visualized in Fig. 4.

Table 1:

dosed $\quad 0$ ppm, measured 2,1 ppm, relative error: -

dosed $250 \mathrm{ppm}$, measured $231 \mathrm{ppm}$, relative error: $7,6 \%$

dosed $500 \mathrm{ppm}$, measured $499 \mathrm{ppm}$, relative error: $0,2 \%$

dosed $1000 \mathrm{ppm}$, measured $1059 \mathrm{ppm}$, relative error: 5,9\%

dosed $2000 \mathrm{ppm}$, measured $2219 \mathrm{ppm}$, relative error: 10,9\%

The analysis errors of these results (less than 11\%) are comparable with those obtained when using original, i.e. non-approximated CTP data for calibration, but, as already mentioned above, the effort for calibration is much lower. Similar good analysis results were also obtained for sensor3 and sensor4.

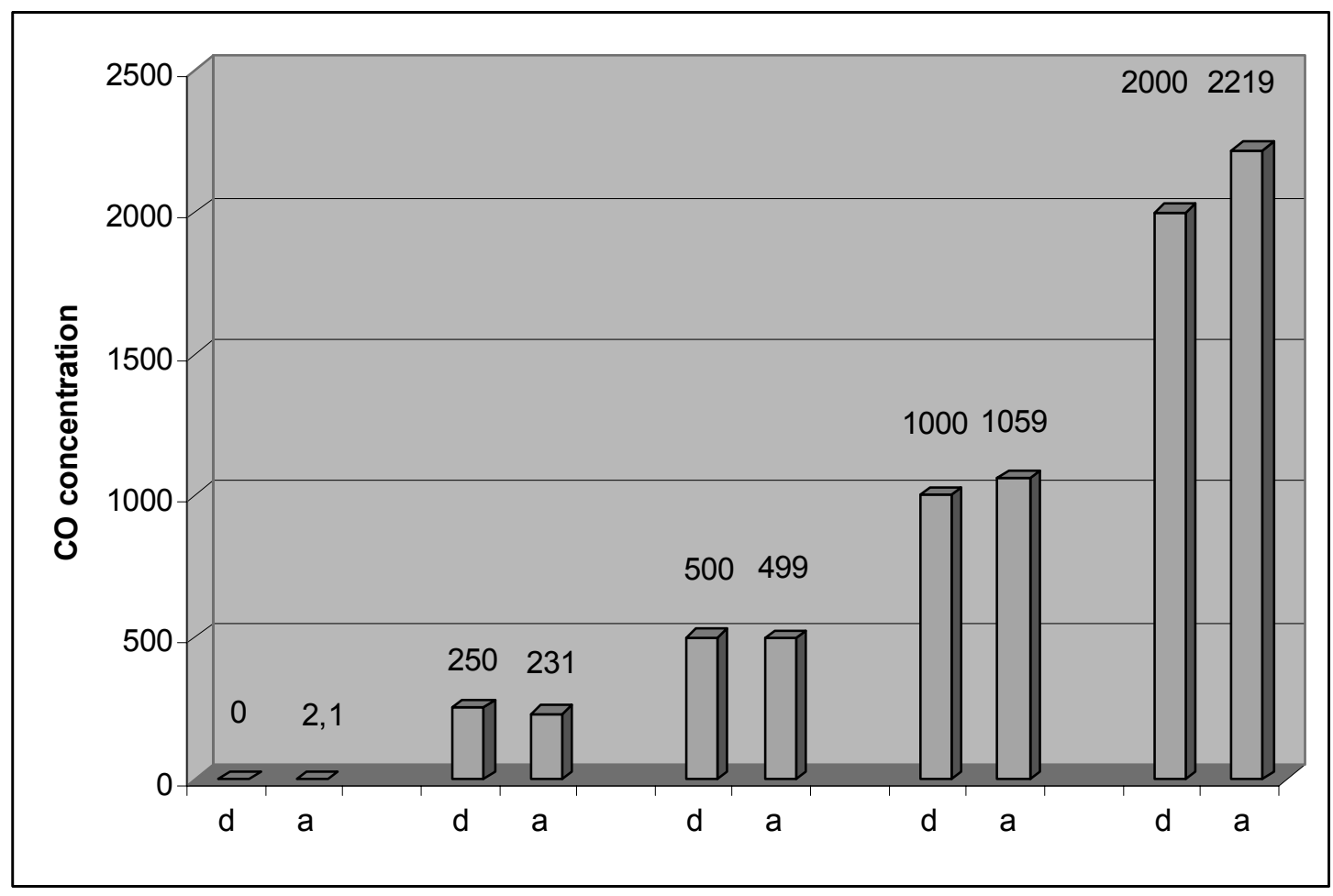

Fig. 4: Comparison of dosed $\mathrm{CO}$ concentrations (d) and analysed $\mathrm{CO}$ concentrations (a) in ppm

\section{Conclusions}

With this new calibration procedure, the very time-consuming and expansive calibration of metal oxide gas sensors can be reduced almost by the factor of five. This enhances the attraction of this type of sensor for low-cost applications substantially. The same procedure can be applied for cost-effective and time-saving recalibration of sensors. The investigations demonstrate that the approximated signal patterns are close to the sampled signal patterns. And these investigations show, that with these approximated signal patterns the concentrations of $\mathrm{CO}$ in synthetic air can be determined very well, using an innovative mathematical evaluation procedure already developed in the past $/ 6 /$. The analysis errors are in all cases less than $11 \%$ which is in the same range of analysis errors obtained when using only sampled signal patterns for calibration $/ 3 /$. 


\section{References}

/1/ Jerger, A.; Kohler, H.; Becker, F.; Keller, H.B.; Seifert, R.

„New applications of tin oxide gas sensors II. Intelligent sensor system for reliable monitoring of ammonia leakages", Sensors and Actuators B, 81(2002) S.301-07

/2/ Frank, K.; Hetznecker, A.; Kohler, H.; Schindler, V.; Schönauer, U.; Arnanthigo, Y.; Falk, D.; Keller, H.B.; Seifert, R.: „Metal oxide gas sensors for field analysis: Novel $\mathrm{SnO}_{2} / \mathrm{La}_{2} \mathrm{O}_{3}$ sensor element for analysis of dissolved toluene/ethanol binary mixtures", Sensor 2005: 12th Internat.Conf., Nürnberg, May 10-12, 2005, Proc.Vol.2 S.207-209, Wunstorf : AMA Service GmbH, 2005

/3/ Frank, K.; Magapu, V.; Schindler, V.; Arnanthigo, Y.; Kohler, H.; Keller, H.B.; Seifert, R.: „Improving the analysis capability of tin oxide gas sensors by dynamic operation, appropriate additives and an advanced evaluation procedure", Sensor 2007 : 13th Internat.Conf., Nürnberg, May 22-24, 2007,Proc.Vol.1 S.139-144, Wunstorf : AMA Service GmbH, 2007

14/ Frank, K. , Magapu, V., Schindler, V., Kohler, H., Keller, H. B., and Seifert, R.: "Chemical Analysis with Tin Oxide Gas Sensors: Choice of Additives, Method of Operation and Analysis of Numerical Signal", Sensor Letters, in press

/5/ Frank, K., Guth, U., Kohler, H.: "Influence of measurement conditions on the sensitivity performance of $\mathrm{SnO}_{2}$ gas sensors operated in the thermo cyclic mode", Sensors and Actuators B, submitted

/6/ Keller, H. B., Seifert, R., Frank, K., Hetznecker, A., Schindler, V., Kohler, H.:

"Mathematical procedure for gas analysis with dynamically operated sensor arrays" 11th Internat.Meeting on Chemical Sensors (ICMS-11), Brescia, I, July 16-19, 2006 